\title{
因HAD
}

ISSN-L: 2530-5115

(1) (1)(2)

BY NO SA

DOI: http://doi.org/10.22585/hospdomic.v4i1.94

\section{Manejo domiciliario del catéter pleural tunelizado permanente: A propósito de un caso}

Home management of permanent tunneled pleural catheter: A case report

Jorge Olea-Soto', Maria J. Soler-Sempere', Salvador Cerveró-Ferragut'

1. Hospital General Universitario de Elche, España.

Correspondencia/Correspondence

Jorge Olea-Soto

joleasoto@hotmail.com

Recibido/Received

05.12.2019

Aceptado/Accepted

06.01 .2020
Conflicto de Intereses/Competing interest Los autores declaran no tener conflicto de intereses permanente: A propósito de un caso. Hosp Domic. 2020;3(1):43-9. 


\section{RESUMEN}

El derrame pleural maligno representa una entidad frecuente en pacientes con enfermedades neoplásicas. Su aparición representa un estadio avanzado de la enfermedad e indica mal pronóstico, y puede conllevar una elevada carga sintomática con el deterioro de la calidad de vida. Aunque su manejo debe individualizarse, en determinados casos, la utilización de catéteres pleurales tunelizados permanentes puede ser una alternativa segura y eficaz para intentar alcanzar un adecuado control de los síntomas.

Presentamos el caso de una paciente diagnosticada en nuestro departamento de un mesotelioma pleural maligno con derrame pleural recidivante, a la que se le colocó un catéter pleural tunelizado puesto que los intentos previos de pleurodesis habían fracasado. Describimos el procedimiento, así como el manejo domiciliario. En este caso, se optó por un abordaje integral con apoyo domiciliario de la Unidad de Hospitalización a Domicilio de nuestro hospital.

En definitiva, el catéter pleural permanente tunelizado representa una alternativa eficaz y segura en el manejo ambulatorio de los pacientes con derrame pleural maligno recidivante y sintomático. Este sistema permite la extracción de líquido pleural en el domicilio del paciente y alcanzar un mayor control de los síntomas respiratorios y una mayor calidad de vida en este grupo de pacientes.

Palabras clave: Derrame Pleural Maligno; Mesotelioma ; Neoplasias; Catéteres; Servicios de Atención de Salud a Domicilio; Servicios de Atención a Domicilio Provisto por Hospital

\section{ABSTRACT}

Malignant pleural effusion represents a frequent entity in patients with neoplastic diseases. Its appearance represents an advanced stage of the disease and indicates a poor prognosis and can lead a high symptomatic burden with the deterioration of the quality of life. Although its management must be individualized, in certain cases, the use of permanent tunneled pleural catheters can be a safe and effective alternative to try to reach an adequate control of symptoms.

We present the case of a patient diagnosed in our department of a malignant pleural mesothelioma with recurrent pleural effusion, to which a tunneled pleural catheter was placed because previous pleurodesis attempts had failed. We describe the procedure, as well as home management. In this case, we chose a comprehensive approach with home support from the Hospital at Home of our hospital.

Keywords: Pleural Effusion, Malignant; Mesothelioma; Neoplasms; Home Care Services; Home Care Services, Hospital-Based 


\section{INTRODUCCIÓN}

El derrame pleural maligno (DPM) es una complicación entre los pacientes con neoplasias. Su aparición representa un estadio avanzado de la enfermedad y mal pronóstico, y suele conllevar una elevada carga sintomática con el deterioro de la calidad de vida de los pacientes (1). Entre las diferentes opciones del manejo paliativo del DPM, el catéter pleural tunelizado permanente representa una alternativa válida en determinados casos seleccionados $(2,3)$.

Esta técnica consiste en la colocación de un catéter tunelizado dentro de la cavidad pleural que permite ser conectado de manera intermitente a una botella de vacío (4). De esta manera, se evita la necesidad de ingresos hospitalarios, la necesidad de toracocentesis evacuadoras de repetición y permite un control de los síntomas derivados del derrame pleural en pacientes oncológicos, ya que el drenaje del líquido pleural se podrá realizar de forma ambulatoria o en el domicilio de los pacientes.

\section{DESARROLLO DE LA EXPERIENCIA}

Presentamos el caso de una paciente de 71 años que presentaba disnea en relación con esfuerzos de mínima intensidad y dolor torácico, síntomas atribuibles a la presencia de un derrame pleural derecho maligno recidivante como consecuencia de un mesotelioma pleural maligno que había sido diagnosticado 18 meses antes. Por este motivo, recibía tratamiento con quimioterapia y se le había practicado, sin éxito, además de varias toracocentesis terapéuticas, hasta tres pleurodesis con talco con la intención de intentar controlar el derrame pleural y disminuir su sintomatología.

A la exploración física presentaba semiología de derrame pleural que ocupaba más de 2/3 del hemitórax derecho. La radiografía de tórax mostró la presencia de derrame pleural derecho masivo (Imagen 1). La tomografía computarizada de tórax reveló progresión de la enfermedad con incremento de las masas a nivel pleural y mediastínico con derrame pleural ipsilateral.

Ante el fracaso de las pleurodesis con talco, y la rápida reacumulación de líquido pleural, se optó por la colocación de un catéter tunelizado permanente en la cavidad pleural con la finalidad de intentar evitar la acumulación de líquido. Posteriormente, la paciente fue derivada a la Unidad de Hospitalización a Domicilio para, además de un abordaje multidimensional de su situación, realizar educación sanitaria del manejo del catéter, curas en el punto de inserción y proceder al drenaje del líquido pleural en el domicilio de la paciente. Durante su seguimiento no ha presentado complicaciones y tampoco ha precisado la realización de nuevas punciones en la pared torácica.

Desde la colocación del catéter tunelizado, debido a la sintomatología de la paciente, precisa de la extracción de líquido pleural cada 3 días con un volumen medio a la semana de $1500 \mathrm{ml}$. 
Imagen 1. Radiografía de tórax antes de la colocación del catéter pleural tunelizado. Se observa un derrame pleural derecho que ocupa hasta 2/3 del hemitórax

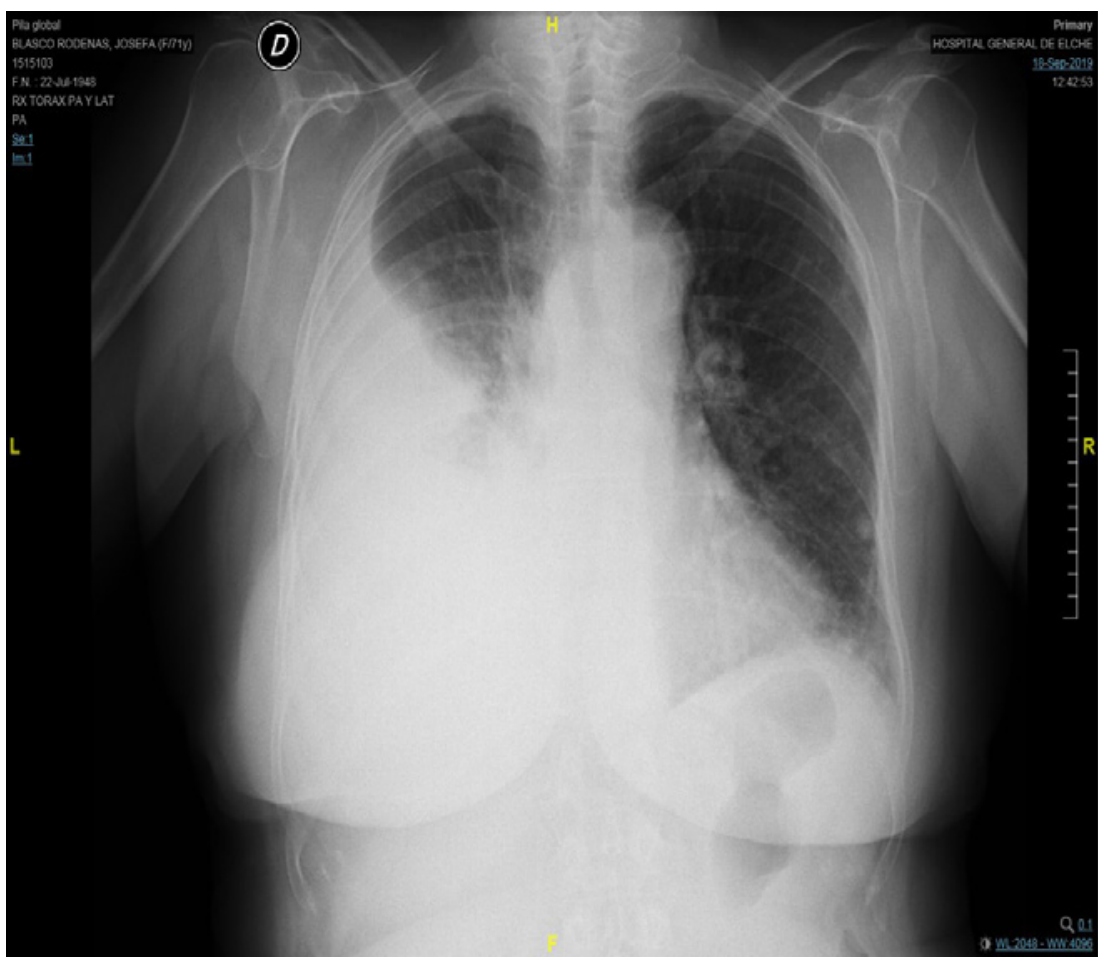

\section{DISCUSIÓN}

El derrame pleural maligno representa hasta el 35 \% de todos los derrames pleurales (5). Generalmente, supone un estadio avanzado de la enfermedad y se asocia a un mal pronóstico. Dependiendo de la cantidad de derrame, el paciente puede presentar sintomatología, siendo la disnea el síntoma más frecuente (3), produciendo en los pacientes un enorme impacto en su calidad de vida y la necesidad de hospitalizaciones (4).

Entre las exploraciones dirigidas al manejo paliativo del DPM, las toracocentesis repetidas, suponen una alternativa, sobre todo si el estado general del paciente es malo y su expectativa de vida limitada. Debido a la elevada recurrencia del DPM, en pacientes con un índice de Karnosfky mayor de 40 y una expectativa de vida superior al mes, la pleurodesis química representa una alternativa, siempre que se demuestre que el pulmón sea reexpansible tras la realización de una toracocentesis terapéutica (5). En los casos en los que la pleurodesis no pueda realizarse o haya fracasado, exista una falta de reexpansión pulmonar tras la evacuación del líquido pleural o en pacientes con corta expectativa de vida, el uso de catéteres tunelizados permanentes representa una alternativa con escasas complicaciones (6). Además, otras de las ventajas del PleurX@ es que se han descrito pleurodesis espontáneas tras la colocación del catéter, hecho que se ha atribuido 
a que al aumentar la frecuencia en el drenaje del líquido pleural favorecería el contacto entre las pleuras y su posterior sínfisis (6).

Las complicaciones reportadas tras su colocación son escasas, siendo las más frecuentes la infección pleural, el dolor en la zona de inserción y la diseminación tumoral en el lugar de la colocación del catéter $(6,7)$.

En nuestro caso, a pesar de que la paciente presentaba un pulmón reexpansible tras el drenaje del líquido pleural, los intentos de pleurodesis con talco fracasaron, y ante la presencia de derrame pleural rápidamente recidivante, se optó por la colocación de un catéter tipo PleurX@. Se trata de un catéter de silicona flexible de $66 \mathrm{~cm}$ de longitud y 15.5 French de grosor, fenestrado que se introduce en la cavidad pleural con anestesia local y se tuneliza a través del tejido celular subcutáneo (Imagen 2). La porción distal a la pared torácica finaliza en una válvula unidireccional de seguridad que sólo se abrirá, permitiendo el drenaje del líquido, cuando es conectada a una botella de vacío de hasta 1 litro de capacidad.

Imagen 2. Extremo distal del catéter pleural tunelizado en la pared torácica de la paciente

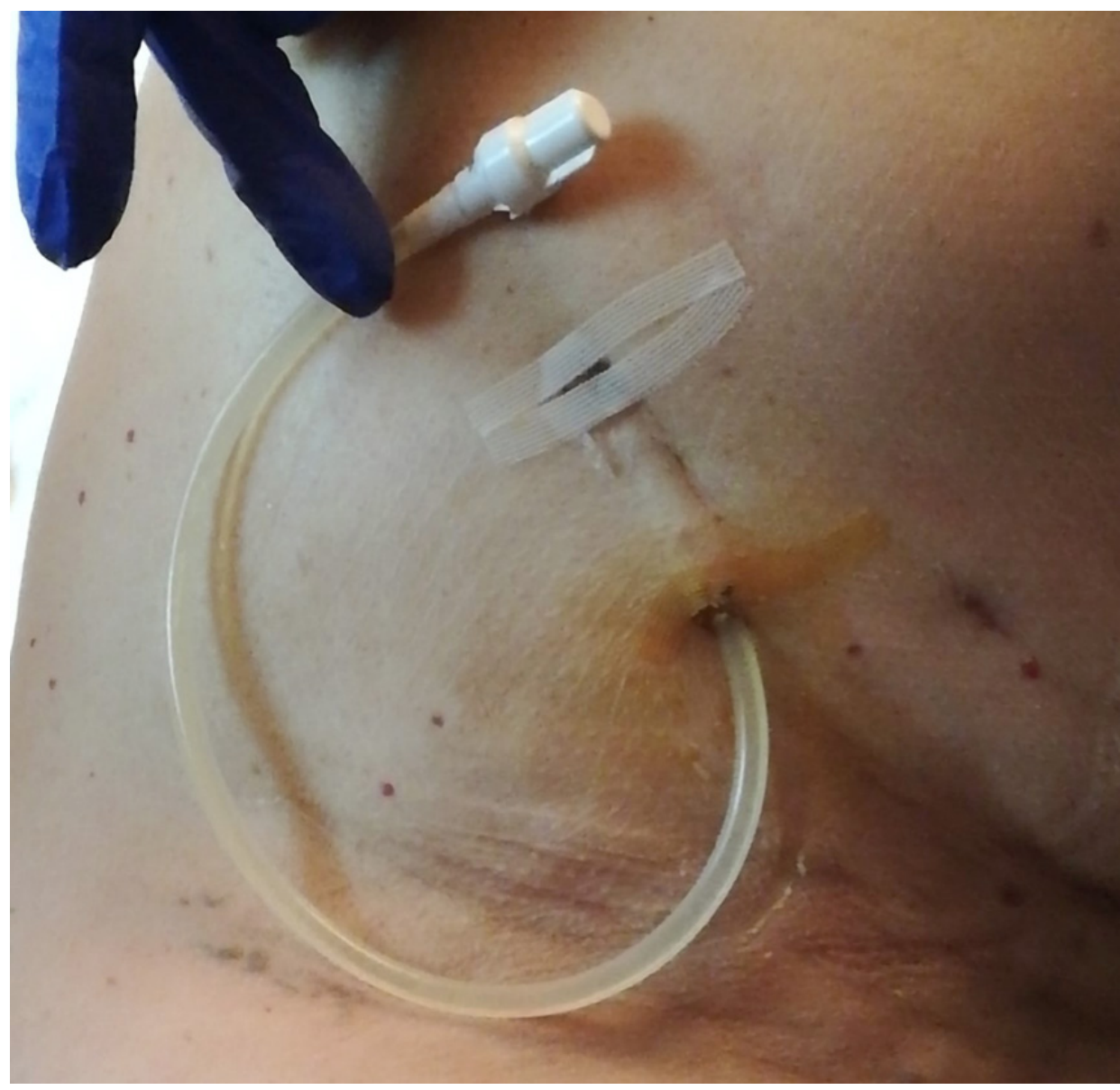


Desde su colocación, la paciente no ha presentado complicaciones a nivel pleural ni en la zona de inserción, y precisa de la evacuación de líquido pleural cada 48-72 horas con una media de 700 $\mathrm{ml}$ de líquido en cada procedimiento (Imagen 3) siendo el limitante a la hora de realizar extracciones más voluminosas la aparición de síntomas por reexpansión pulmonar.

Imagen 3. Drenaje del líquido pleural en el domicilio de la paciente. Se aprecia el líquido en la botella de vacío

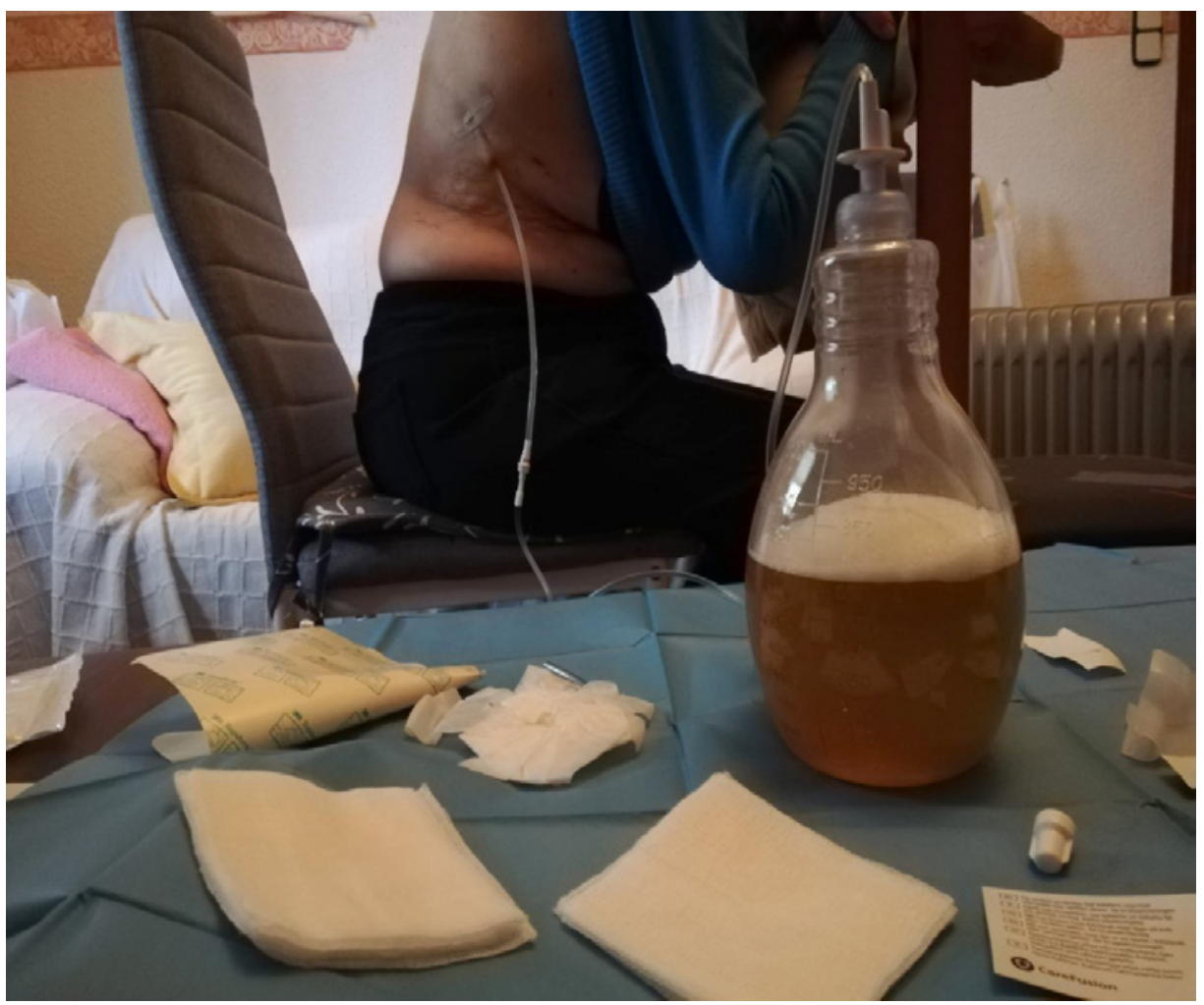

Este procedimiento representa una alternativa para el manejo del derrame pleural maligno, diseñado para que el paciente, instruido en la técnica, pueda drenarse el líquido pleural en su propio domicilio a medida que aparezcan los síntomas. De esta forma se evitarán traslados a centros hospitalarios permitiendo una mejoría en la calidad de vida $(8,9)$. Aunque es una técnica diseñada para que el propio paciente pueda realizarla, consideramos que al tratarse de pacientes con enfermedad maligna avanzada y en muchos casos con un deterioro funcional significativo, se pueden beneficiar de la participación de equipos de soporte domiciliario para el manejo integral del paciente, ya que se debe tener en cuenta que los síntomas que pueden presentar no siempre serán atribuibles, de forma exclusiva, a la afectación pleural. 


\section{CONCLUSIONES}

El catéter pleural permanente tunelizado representa una alternativa eficaz y segura en el manejo ambulatorio de los pacientes con derrame pleural maligno recidivante y sintomático. Este sistema permite la extracción de líquido pleural en el domicilio del paciente y alcanzar un mayor control de los síntomas respiratorios, así como una mejor calidad de vida en este grupo de pacientes.

\section{BIBLIOGRAFÍA}

1. Porcel JM, Gasol A, Bielsa S, Civit C, Light RW, Salud A. Clinical features and survival of lung cancer patients with pleural effusions. Respirology. 2015; 20(4):654-9. DOI: 10.1111/resp.12496; PMID: 25706291

2. Álvarez FV, Trueba IM, Sanchis JB, López-Rodó LM, Rodríguez Suárez PM, de Cos Escuín JS, et al. Recommendations of the Spanish Society of Pneumology and Thoracic Surgery on the diagnosis and treatment of non-small-cell lung cancer. Arch Bronconeumol. 2016;52(Suppl 1):2-62. DOI: 10.1016/S0300-2896(16)30198-3; PMID: 27389767

3. Feller-Kopman DJ, Reddy CB, DeCamp MM, Diekemper RL, Gould MK, Henry T, et al. Management of Malignant Pleural Effusions - An Official ATS/STS/STR Clinical Practice Guideline. Am J Respir Crit Care Med. 2018;198(7):839-49. DOI: 10.1164/rccm.201807-1415ST; PMID: 30272503

4. Cano Hoz M, Priede Díaz L, Carrascosa Porras M. Tratamiento ambulatorio del derrame pleural maligno: aportación de un caso. Med Paliat. 2012;19(1):3-9. DOI:10.1016/j.medipa.2010.12.002

5. Villena Garrido V, Cases Viedma E, Fernández Villar A, de Pablo Gafas A, Pérez Rodríguez E, Porcel Pérez JM, et al. Recommendations of diagnosis and treatment of pleural effusion: Update. Arch Bronconeumol. 2014;50(6):235-49. DOI: 10.1016/j.arbres.2014.01.016; PMID: 24698396

6. Cases E, Seijo L, Disdier C, Lorenzo MJ, Cordovilla R, Sanchis F, et al. Uso del drenaje pleural permanente en el manejo ambulatorio del derrame pleural maligno recidivante. Arch Bronconeumol. 2009;45(12):591-6. DOI: 10.1016/j.arbres.2009.09.009; PMID: 19879036

7. Kaifi JT1, Toth JW, Gusani NJ, Kimchi ET, Staveley-O'Carroll KF, Belani CP, et al. Multidisciplinary management of malignant pleural effusion. J Surg Oncol. 2012;105(7):731-8. DOI: 10.1002/ jso.22100; PMID: 21960207

8. Putnam JB Jr, Walsh GL, Swisher SG, Roth JA, Suell DM, Vaporciyan AA, et al. Outpatient management of malignant pleural effusions. Ann Thorac Surg. 2000;69(2):369-75. DOI: 10.1016/ s0003-4975(99)01482-4; PMID: 10735665

9. Páez-Codeso F, Dorado-Galindo A, Jiménez-Fernández DM, Bermejo-Casero E, Ruarte-Naranjo C, González-Angulo GE, et al. Tratamiento paliativo ambulatorio de disnea con catéter tunelizado en derrame pleural maligno: Estudio descriptivo. Neumol Cir Torax. 2017;76(4): 308-14. 